\title{
Some Characteristics of the E-ELT Sodium LGSs
}

\author{
N. Moussaoui*, F. Djaidri and F. Zohra OuAnoufi \\ University of Sciences and Technology Houari Boumediene (USTHB), Faculty of Physics, \\ Quantum Electronic Laboratory, Algiers, Algeria
}

\begin{abstract}
Adaptive optics systems require a source of light to measure and to correct wave-front distortions. This reference source of light must be in the field of view of the astronomical subject observed by the ground based telescope. There are not enough natural stars that could be used by the adaptive optics systems. In order to insure a full sky coverage of adaptive optics system beam tuned to the sodium $D_{2}$ line at $589 \mathrm{~nm}$. The characteristics of the sodium laser guide stars depend on the laser beam intensity, the laser beam polarization, the properties of the mesospheric sodium layer, the atmospheric transmission, and the geomagnetic field characteristics. For the properties of the mesospheric sodium layer we are interested in this study on the sodium layer column abundance, the centroid height, the sodium concentration, and the layer thickness. This part of our work is based on measurements of the mesospheric sodium layer characteristics which cover a long period at latitude $23^{\circ}$ south, in São José dos Compos, São Paulo, Brazil. This latitude is approximately the same as for the European Extremely Large Telescope site (Paranal, Chile), which is located at latitude $24^{\circ}$ south. The geomagnetic field effect is deduced from semi-empirical model realized by the Star Fire Optical Rang team and generalized by the European Southern Observatory Laser Guide Star Group. This work presents some characteristics of the sodium laser guide stars that could be created for the European-Extremely Large Telescope.
\end{abstract}

DOI: 10.12693/APhysPolA.137.496

PACS/topics: adaptive optics, extremely large telescope, sodium-LGSs

\section{Introduction}

For optimal operation, the adaptive optics (AO) system requires artificial guide stars. The AO-sodium laser guide stars (AO-LGSs) combination provides full coverage of the sky. The process used to produce LGSs is the resonant excitation of the sodium atoms [1-3]. The laser used is tuned to the wavelength of the $D_{2}$ line of the sodium atoms. The laser beam projected toward mesospheric layer then excites a sodium column. Optical pumping of atomic sodium with circularly polarized light can increase the effective absorption cross-section theoretically by a factor of more than 2 compared to non-polarized excitation of the $F=2$ hyperfine ground state $[4,5]$. The main goal of this work is to show the impact of the geomagnetic field and the sodium layer on the AO-LGSs of the E-ELT.

\section{Sodium atoms excitations}

\subsection{Sodium excitation}

Atomic sodium has a total of 11 electrons with a single valence electron outside closed shells. The complete term symbol of the ground state is $1 s^{2} 2 s^{2} 2 p^{6} 3 s^{2} S_{1 / 2}$, and for the first excited state, it is $1 s^{2} 2 s^{2} 2 p^{6} 3 s^{2} P_{1 / 2,3 / 2}$. The interaction of the magnetic moment of the electron with the magnetic field associated with the orbital motion of the electron leads to the energy level splitting within

*corresponding author; e-mail: nmoussaoui@usthb.dz the first excited state, $3^{2} P_{1 / 2}, 3^{2} P_{3 / 2}$. The two states are separated in energy by about $520 \mathrm{GHz}$. The transitions between the upper state $3^{2} P_{3 / 2}$ and the ground state $3^{2} S_{1 / 2}$ cause the sodium $D_{2}$ emission or absorption at $589.2 \mathrm{~nm}$, while the transitions between the lower state $3^{2} P_{1 / 2}$ and the ground state cause the sodium $D_{1}$ emission or absorption at $589.6 \mathrm{~nm}$. The total electronic angular momentum of the ground state and first excited state are $J=1 / 2$ and $J=1 / 2,3 / 2$, respectively. Naturally occurring sodium is composed virtually $100 \%$ of one isotope, which has a nuclear spin of $I=3 / 2[6,7]$.

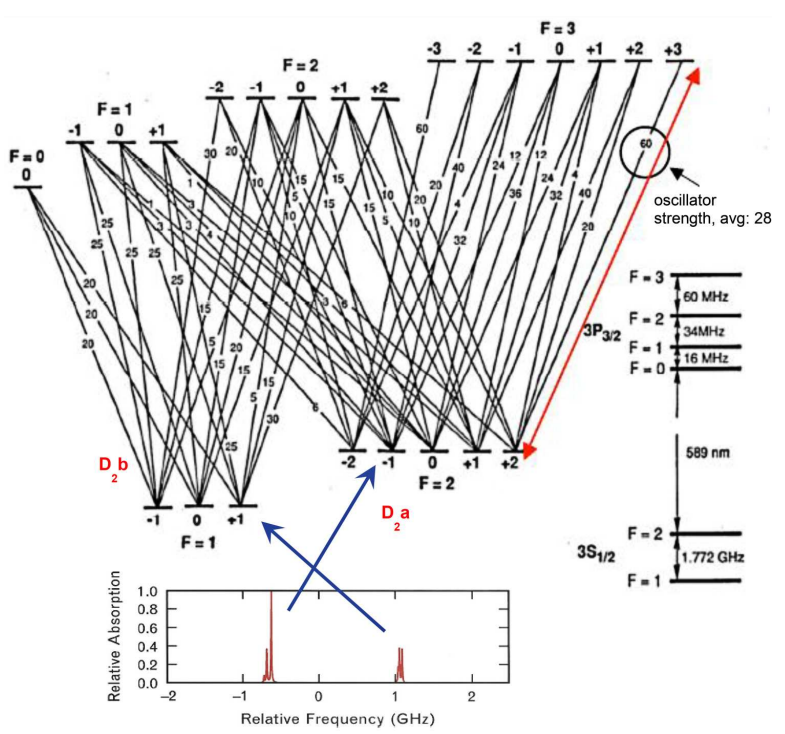

Fig. 1. Sodium atom's transitions (graphics by Ungar [8]). 
The interaction of an electron with the nuclear magnetic moment leads to the hyperfine structures associated with the ground and excited states of the sodium atom. The total angular momentum quantum number $F$ is the sum of nuclear spin $I$ and the electron spin $J$. The resulting total angular momentum quantum numbers are $F=1,2$ for the sodium ground state $3^{2} S_{1 / 2}, F=1,2$ for the $3^{2} P_{1 / 2}$ excited state, and $F=0,1,2,3$ for the $3^{2} P_{3 / 2}$ excited state. The energy difference between the hyperfine states $F=2$ and $F=1$ in the ground state is $1.772 \mathrm{GHz}$. The energy separation for the hyperfine splitting in the $J=1 / 2$ state of the first excited state is 188.6 MHz, while the energy separations of the $J=3 / 2$ state are $15.8,34.4$, and $58.3 \mathrm{MHz}$ for the four hyperfine states with $F=0,1,2,3$, respectively.

The sodium $D_{2}$ transitions are chosen for the generation of sodium artificial stars because they have total line strength greater by a factor of two than the $D_{1}$ transitions (Fig. 1). Furthermore, the $D_{2}$ transitions have much better optical-pumping characteristics, which results in more efficient excitation of the sodium atoms in the mesospheric sodium layer. After many absorptiondecay cycles, atoms that start in the $F=2$ level end up in the $F=2, M_{F}=2$ state. This state has the largest cross-section $[9]$.

\subsection{Geomagnetic field effect}

The magnetic quantum number $M_{F}$ is defined with respect to the quantization axis chosen to describe the interaction of the atom with the laser field. In the case of a circularly polarized laser field, this axis is chosen to be along the direction of propagation of the field.

The weak Earth static magnetic field at the mesosphere cannot cause transitions between states of different energy, and, in particular, it cannot cause transition between states with different values of $F$. It will cause transitions only between states with different $M_{F}$ and the same $F$. The geomagnetic field acts on the redistribution of magnetic substates, which would reduce the degree of optical pumping and, in the worst case, eliminate the advantage of using circularly polarized laser radiation. To understand this one has to solve the density-matrix equations [5]. Numerical solutions of these equations are demanding. The simplest nontrivial case, $F=1$, has been solved numerically [9].

For our sodium transition, the occupation probabilities of the $3^{2} S_{1 / 2}(F=2, M=2)$ state and of the $3^{2} P_{3 / 2}$ $(F=3, M=3)$ state were calculated [10] as a function of the angle between the laser beam propagation direction and the Earth magnetic field, and time (Fig. 2).

\section{Impact of the geomagnetic field on the sodium LGS}

The geomagnetic field does not have the same intensity and the same orientation (declination and inclination) all over the world. To estimate this effect on the LGS
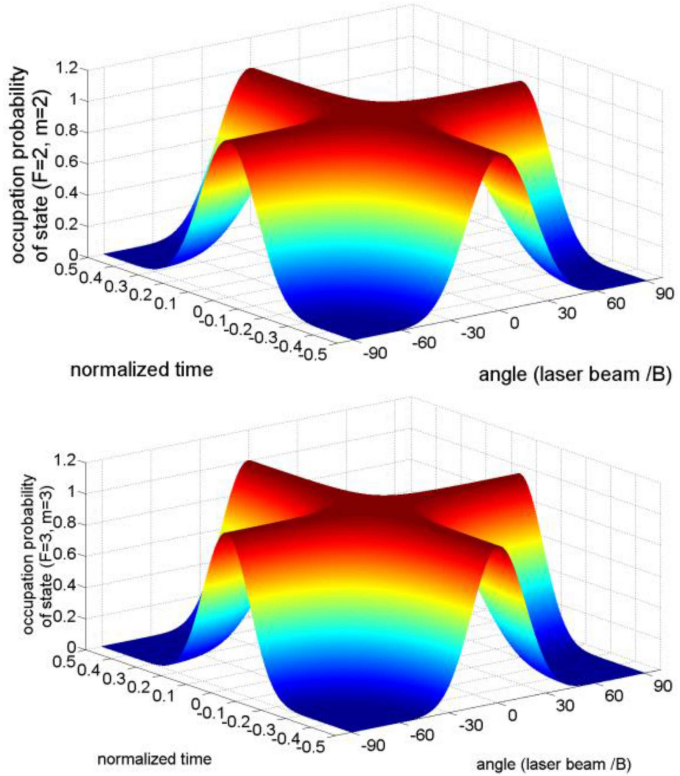

Fig. 2. Occupation probabilities as function of time and angle between geomagnetic field and laser beam.

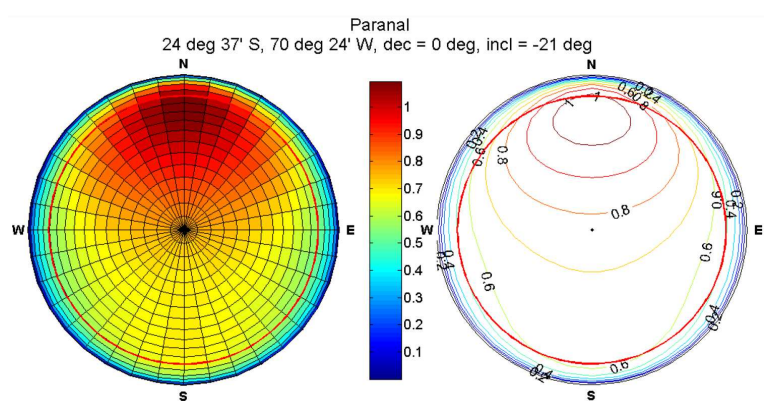

Fig. 3. Geomagnetic effect on the sodium LGS return flux.

intensity we have to know the angle it makes with the laser beam propagation direction for each telescope.

The impact of the Earth magnetic field on the brightness of the LGS depends on the angular distance $\theta$ between the laser beam propagation and the direction of the magnetic field lines. The expression for the magnetic field brightness dependence factor for circular polarization is proportional to the occupation probability the atomic states, following [10].

The optical pumping of the mesospheric sodium atoms with circularly polarized laser light is most efficient when the beam is parallel to the geomagnetic field lines. As northern Chile (site of the E-ELT) is located relatively close to the magnetic equator, this direction lies close to the horizon. For single-frequency lasers, the variation of return flux, when moving the beam from the direction parallel to the field lines to orthogonal, is about a factor of $2.25[11,12]$. For other laser formats, we expect smaller factors. 
We propose that the LGS laser power must be budgeted for the direction of minimum return, since we need to be able to observe in any direction above 30 degrees altitude with LGS-AO. Therefore, we disregard the return benefits of optical pumping in the calculation of the required laser power.

Figure 3 presents polar plot of the relative radiance enhancement of the sodium LGS due to optical pumping with circularly polarized laser beams in the presence of the geomagnetic field for the E-ELT telescope (Paranal, Chile).

\section{Conclusion}

The E-ELT will constitute a real revolution in the astronomical observation from the ground. Its angular resolution is 15 times greater than the HST (Hubble Space Telescope) resolution. The use of its LGSs-AO system will reduce the cone effect drastically. Therefore, the quality of the observation from the ground could be compared to the observations from space.

The magnetic field in northern Chile (site of the E-ELT), is quite weak ( $B=0.23 \mathrm{G})$, and hence optical pumping may be disrupted less than at other sites such as the SOR in Albuquerque, New Mexico $(B=0.51 \mathrm{G})$ or in Hawaii $(B=0.35 \mathrm{G})$ for example $[13,14]$.

\section{References}

[1] N. Moussaoui, B.R. Clemesha, R. Holzlöhner, D.M. Simonich, D. Bonaccini Calia, W. Hackenberg, P.P. Batista, Astron. Astrophys. A31, 511 (2010).

[2] K. Wei, Min Li, Shan-Qiu Chen, et al., Res. Astron. Astrophys. 16, 183 (2016).
[3] R. Foy, A. Labeyrie, Astron. Astrophys. 152, L29 (1985).

[4] P.W. Milonni, R.Q. Fugate, J.M. Telle, J. Opt. Soc. Am. A 15, 217 (1998).

[5] K. Gottfried, Quantum Mechanics, Benjamin, Reading (MA) 1977, p. 269.

[6] M. Özer, M. Orhan, Int. J. Computat. Exp. Sci. Eng. (IJCESEN) 1, 19 (2015).

[7] M. Orlić, I.J. Badovinac, N. Orlić, Int. J. Computat. Exp. Sci. Eng. (IJCESEN) 1, 36 (2015).

[8] P.J. Ungar, D.S. Weiss, E. Riis, S. Chu, J. Opt. Soc. Am. B 6, 11 (1989).

[9] J.R. Morris, J. Opt. Soc. Am. A 11, 832 (1994).

[10] N. Moussaoui, R. Holzlöhner, W. Hackenberg, D. Bonaccini Calia, Astron. Astrophys. 501, 793 (2009).

[11] J.D. Drummond, S.J. Novotny, C.A. Denman, P.D. Hillman, J.M. Telle, M.L. Eickhoff, R.Q. Fugate, "The Sodium LGS Brightness Model over the SOR", in: AMOS Technical Conf., 2007.

[12] C.A. Denman, G. Moore, J.D. Drummond, M.L. Eickhoff, P.D. Hillman, J.M. Telle, S.J. Novotny, R.Q. Fugate, "Two-Frequency Sodium Guide Star Excitation at the Starfire Optical Range", in: CFAO, Workshop, 2006 .

[13] C.A. Denman, J.D. Drummond, M.L. Eickhoff, R.Q. Fugate, P.D. Hillman, S.J. Novotny, J.M. Telle, Proc. SPIE 6272, 62721L, (2006).

[14] J.E. Kibblewhite, Proc. SPIE 7015, 70150M (2008). 